\title{
Grouping behaviour and decision making in road tunnels evacuation in smoke conditions Experimental approach
}

\author{
Jarosław Wąs*1, Jakub Porzycki ${ }^{1}$, Natalia Schmidt-Polończyk ${ }^{2}$ \\ ${ }^{1}$ AGH University of Science and Technology \\ Institute of Applied Computer Science \\ Kraków, Poland \\ ${ }^{2}$ AGH University of Science and Technology \\ Faculty of Mining and Geoengineering \\ Kraków, Poland \\ *e-mail : jarek@agh.edu.pl
}

Faculty of Electrical Engineering, Automatics, Computer Science and Biomedical Engineering

\begin{abstract}
We have performed a set of evacuation experiments in a road tunnel. In each experiment pedestrians were gathered in a bus, the bus was stopped in the tunnel, next the tunnel was filled with artificial smoke and pedestrians had to evacuate. We compared evacuation times and behaviours for different levels of visibility, defined by extinction coefficient Cs range.
\end{abstract}

Keywords: Evacuation experiments, grouping behaviour, decision making, artificial smoke

\section{Introduction}

It should be stressed that the experimental approach is very important in evacuation modelling. This makes it possible to estimate behaviours of evacuees in different conditions. However, organization of such tests is not an easy task due to ensuring safety of participants, the high costs of such projects and support required from different services like the fire brigade and ambulance service. One has to select only some scenarios, and the "surprise effect" can only be observed once for each group of participants, as in subsequent trials one can identify the influence of the learning effect on pedestrian behaviour. Anyway, experimental results are necessary both for a better understanding of evacuees' behaviour as well as in the calibration and validation of computer models of evacuation or risk assessment.

\section{Related works}

There are a number of works on tunnels evacuation, as well as pedestrian behaviour and decision making.

Seike et al. in [1] analysed influence of the extinction coefficient on walking speed of individual pedestrians in a tunnel. Nilsson et al. in [2] took into account behaviour of motorists in a road tunnel during an unannounced evacuation. Wang et al. in [3] presented experimental research of an evacuation of pedestrians walking blindfolded. Nilsson et al. discussed in [4] how research can be connected to large infrastructure tunnel projects taking into account fire safety issues.

The simultaneous modelling approach is present in research on evacuation dynamics. Ronchi et al. [5] presented virtual experiments on evacuation trajectories, while Lovreglio et al. [6] introduced an evacuation decision model taking into account perceived risk, social influence and behavioural uncertainty. Ronchi et al. in [7] analysed walking speeds in smoke in evacuation models.

\section{Description of experiments}

In this paper we take into consideration the decision-making process during evacuation in tunnels, as well as grouping behaviours. We have performed a set of full-scale evacuation experiments with artificial smoke in the "Emilia" road tunnel located in the south of Poland.

We gathered 50 participants in a bus, (Fig 1 and Fig 2). The group consists of 34 males and 16 females. The average age is 21 years $(21.32,=1: 477$,$) age span is in this case 19$ to 24 years. In the experiment 1 , the participants have not received any instructions as to the purpose of the experiment 
how to behave in case of smoke, infrastructure of the tunnel namely the location of the evacuation cross - passages as well as the position of the bus in the tunnel. The bus has been directed into the tunnel without stopping, in order to ensure an element of surprise among the participants. The position of the bus in this experiment has been in the middle of the tunnel, just like in the experiments 2 and 3 .

Artificial smoke generators were used in order to investigate grouping behaviours [8] and the decision making process for different visibility levels. In each case a vehicle with pedestrians was stopped in a tunnel and artificial smoke was generated around them (Fig 1). Then the pedestrians initialized evacuation from the vehicle and the smoke filled road tunnel. In all cases, the driver was instructed to be passive, namely not to instruct passengers about escape routes and further proceedings, or to get up form seats and evacuate.

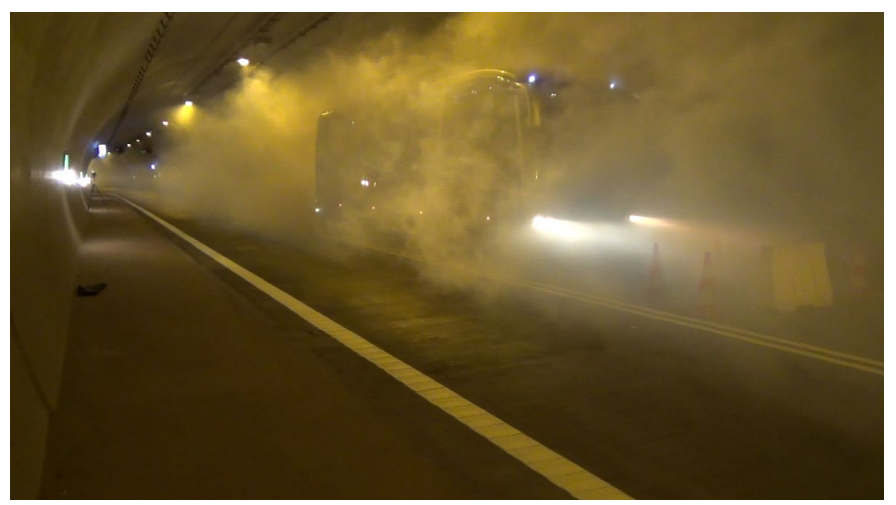

Fig 1. Our coach with participants in the "Emilia" tunnel filled with artificial smoke - pre-movement phase of evacuation

During experiments participants evacuated from the main tunnel (road tube), filled with artificial smoke, to the evacuation tunnel, situated parallel to the road tube.

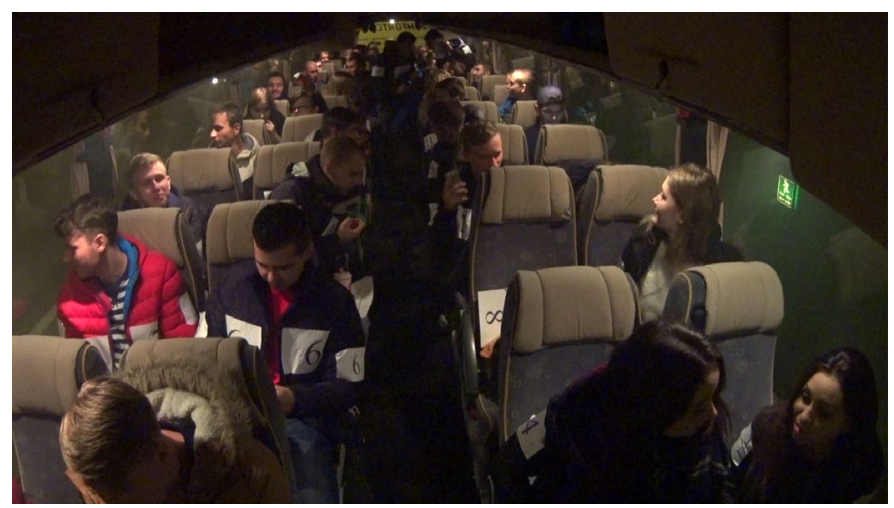

Fig 2 Participants in the coach before experiments in the "Emilia tunnel"

As scientific methodology we applied analyses based on video recordings with standard and infrared cameras, direct observations, segment measurements using UHF technology and questionnaires filled by participants. Participants had assigned numbers, cards with numbers were placed on their clothes (Fig 2).

\section{Sample results}

In the "Emilia" tunnel we performed the following experiments using different range of visibility coefficient Cs, namely the extinction coefficient based on the Lambert-Beer equation [1]. 
Tab 1. List of experiments with different levels of visibility, tunnel familiarity of evacuated group of participants and expressed task.

\begin{tabular}{|l|l|l|l|}
\hline $\begin{array}{l}\text { Experiment } \\
\text { number }\end{array}$ & $\begin{array}{l}\text { Visibility } \\
\text { Cs - range }\end{array}$ & $\begin{array}{l}\text { Tunnel } \\
\text { Familiarity }\end{array}$ & $\begin{array}{l}\text { Task for } \\
\text { pedestrians }\end{array}$ \\
\hline 1 & $0.1-0.2 \mathrm{~m}^{-1}$ & No & $\begin{array}{l}\text { No expressed } \\
\text { task }\end{array}$ \\
\hline 2 & $0.4-0.5 \mathrm{~m}^{-1}$ & Yes & To evacuate \\
\hline 3 & $0.8-0.9 \mathrm{~m}^{-1}$ & Yes & $\begin{array}{l}\text { To achieve the } \\
\text { best personal } \\
\text { time }\end{array}$ \\
\hline
\end{tabular}

Regarding movement speed we observed different velocities in the main tunnel during our experiments. Taking into account pedestrian flow in smoke during three experiments in the "Emilia" tunnel we observed the following distributions of velocities (Fig 3 and Tab 2). In the first experiments the observed speed was relatively low, because it was a new situation for participants and they had to make decisions about evacuation path. Despite the increase of smoke in subsequent experiments, the speed of the first persons increased markedly - "learning effect" was observed.

It should be stressed that in the third experiment we asked participants for to achieve potentially the best individual evacuation time. As a result we observed more competitive behaviour, however due to lower level of visibility the velocities were lower than in experiment 3 .

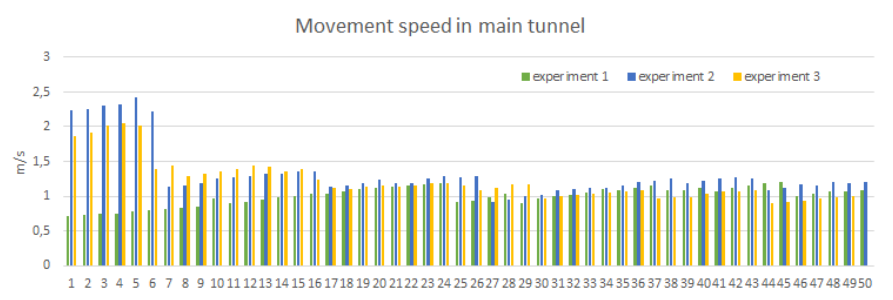

Fig 3. Movement speed of particular pedestrians in the "Emilia" tunnel experiments in three consecutive experiments.

Tab 2 Movement speed for experiments 1-3 - overall statistics.

\begin{tabular}{|l|l|l|l|l|}
\hline $\begin{array}{l}\text { Experiment } \\
\text { Number }\end{array}$ & Min. & Max. & Mean & $\begin{array}{l}\text { Std. } \\
\text { deviation }\end{array}$ \\
\hline 1 & 0.895 & 1.211 & 1.056 & 0.083 \\
\hline 2 & 0.917 & 2.422 & 1.321 & 0.375 \\
\hline 3 & 0.893 & 2.044 & 1.221 & 0.295 \\
\hline
\end{tabular}

Additionally we checked the time spans between the first and the last evacuees at the following checkpoints: the bus doors and exits from main tunnel (smoke filled area).

Tab 3. Comparison of time span between the first and the last evacuee at each checkpoint for experiments 1-3

\begin{tabular}{|l|l|l|}
\hline $\begin{array}{l}\text { Experiment } \\
\text { Number }\end{array}$ & $\begin{array}{l}\text { Bus door } \\
\text { Checkpoints }\end{array}$ & $\begin{array}{l}\text { Main tunnel exits } \\
\text { checkpoints }\end{array}$ \\
\hline 1 & First: $35.81 \mathrm{~s}$ & First: $84.21 \mathrm{~s}$ \\
& Last: $130.92 \mathrm{~s}$ & Last: $162.73 \mathrm{~s}$ \\
\hline 2 & First: $5.39 \mathrm{~s}$ & First: 20.9 \\
& Last: $85.03 \mathrm{~s}$ & Last: $113.86 \mathrm{~s}$ \\
\hline 3 & First: $2.92 \mathrm{~s}$ & First: $21.16 \mathrm{~s}$ \\
& Last: $67.65 \mathrm{~s}$ & Last: $102.04 \mathrm{~s}$ \\
\hline
\end{tabular}

As we can see in Tab 3, familiarity with a situation and previous experiment cause significantly shorter evacuation times between the second and the first experiment. We observed very interesting results when we compared the results of experiments 3 and 2. Limited visibility (reduction from 0.4- 
$0.5 \mathrm{~m}-1$ to $0.8-0.9 \mathrm{~m}-1$ ) should potentially cause longer evacuation time, however one can notice the effect of mobilization due to increasing level of competitiveness.

Regarding decision making, pedestrians tried to identify the nearest evacuation exit. The tunnel is equipped with evacuation signs, which point out the distance to the nearest exit (Fig 4). This information was important in the context of decision making.

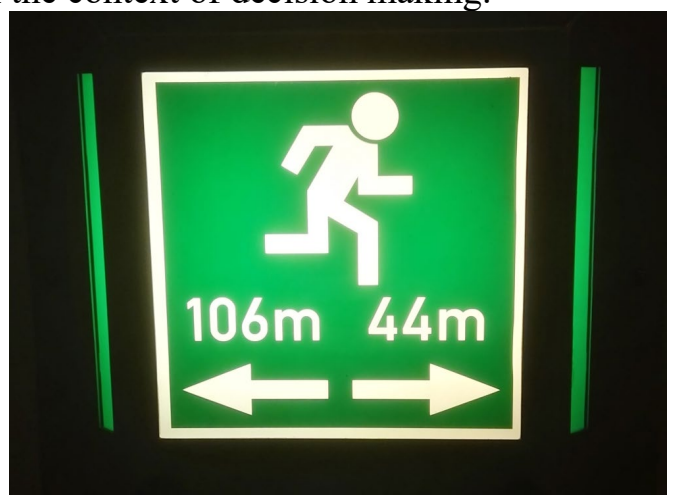

Fig 4. Evacuation signs with the distance to evacuation exits from the main tunnel

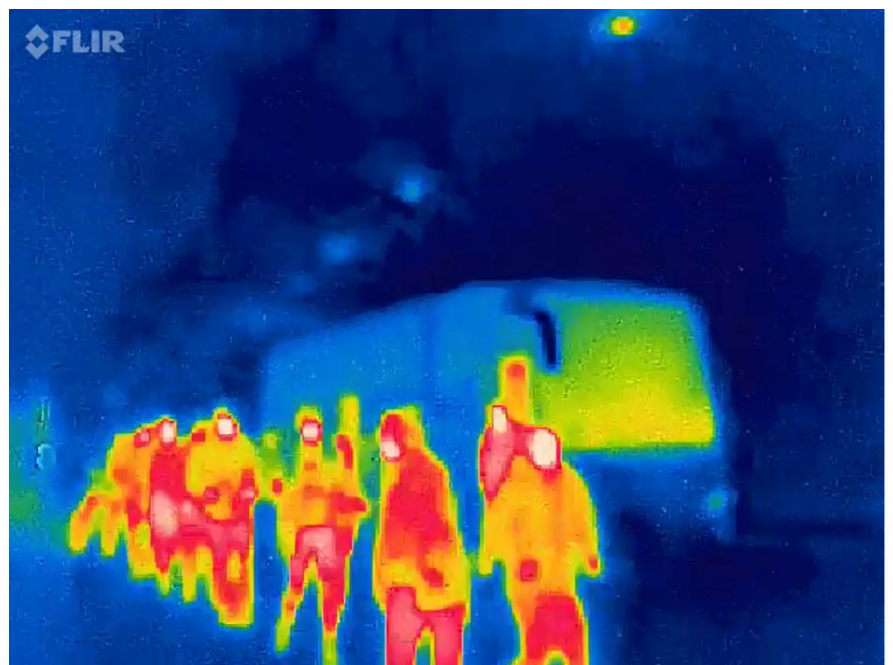

Fig .5 Pedestrians during evacuation in experiment 3. Double line patterns are observed.

According to different levels of visibility we identified different grouping behaviours and different patterns formed by evacuees. During evacuation of a group in low and moderate level of smokiness (when Cs $<0.5 \mathrm{~m}-1$ ) we observed multi-line patterns created by pedestrians. Only the first group of passengers engaged in active decision making, while during heavy smokiness Cs $>0.7 \mathrm{~m}-1$ we observed characteristic double-line patterns (Fig 5.).

\section{Conclusions}

Experiments show two potentially dangerous behaviours: group/leader following and delaying of the decision to begin the evacuation. Despite signals to begin evacuation (like appearance of smoke and siren alarm), participants remain in their seats until they hear a voice message. Following the leader and following the group behaviour occurs in both cases: decision on evacuation start and path choice. Evacuees have a strong tendency to follow the group, if only they can see each other.

On the one hand we have gathered statistics on pedestrians' velocities in a smoke filled tunnel (the first phase of the evacuations) and an evacuation tunnel without smoke (the second phase of evacuation). This makes it possible to compare velocities in smoke and the desired velocity for particular pedestrians. 
Additionally, in questionnaires participants pointed out different issues related to decision making, well-being during the evacuation, and an assessment of technical infrastructure in the tunnel and so on.

\section{Acknowledgements}

This research is supported by grants no 11.11.120.859 and 15.11.100.078.

\section{References}

[1] Miho Seike, Nobuyoshi Kawabata, Masato Hasegawa, Experiments of evacuation speed in smokefilled tunnel, Tunnelling and Underground Space Technology, Volume 53, 2016, Pages 61-67, https://doi.org/10.1016/j.tust.2016.01.003.

[2] Daniel Nilsson, Maria Johansson, Håkan Frantzich, Evacuation experiment in a road tunnel: A study of human behaviour and technical installations, Fire Safety Journal, Volume 44, Issue 4, 2009, Pages 458-468

[3] Shu-jie Wang, W. g. Song and W. Lv, "Moving characteristics of "blind" people evacuating from a room," 17th International IEEE Conference on Intelligent Transportation Systems (ITSC), Qingdao, 2014, pp. 548-553

[4] D. Nilsson, H. Frantzich, E. Ronchi, K. Fridolf, A. L. Walter, and H. Modig.Integrating evacuation research in large infrastructure tunnel projects - experiences from the Stockholm bypass project. Fire Safety Journal, 2017.

[5] Enrico Ronchi, Max Kinateder, Mathias Müller, Michael Jost, Markus Nehfischer, Paul Pauli, Andreas Mühlberger, Evacuation travel paths in virtual reality experiments for tunnel safety analysis, Fire Safety Journal, Volume 71, 2015, Pages 257-267, https://doi.org/10.1016/j.firesaf.2014.11.005.

[6] R. Lovreglio, E. Ronchi, and D. Nilsson. An evacuation decision model based on perceived risk, social influence and behavioural uncertainty. Simulation Modelling Practice and Theory, 66:226 242, 2016

[7] E. Ronchi, S. M. V. Gwynne, D. A. Purser, and P. Colonna. Representation of the impact of smoke on agent walking speeds in evacuation models. Fire Technology, 49(2):411-431, 2013.

[8] Jarosław Wąs, Experiments on Evacuation Dynamics for Different Classes of Situations, Pedestrian and Evacuation Dynamics 2008 Springer Berlin Heidelberg, pages $=" 225$ 